\title{
ORLIB: A Computer Code that Produces Orie-Energy Group, Time- and Spatially-Averaged Neutron Cross Sections
}

\author{
J. A. Blink \\ R. E. Dye \\ J. R. Kimlinger
}

Manuscript Date: December 1981

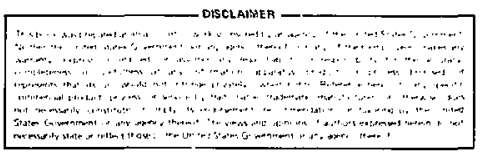

LAWRENCE LIVERMORE LABORATORY

University of California - Livermore, California $\bullet 94550$ 


\title{
ORLIB: A Computer Code that Produces One-Energy Group, Time- and Spatially-Averaged Neutron Cross Sections
}

\begin{abstract}
Calculation of neutron activation of proposed fusion reactors requires a library of neutron-activation cross sections. One such library is $\mathrm{ACTL},{ }^{1}$ which is being updated and expanded by Howerton. ${ }^{2}$ If the energy-dependent neutron flux is also known as a function of location and time, the buildup and decay of activation products can be calculated. In practice, hand calculation is impractical without energy-averagel cross sections because of the large number of energy groups. A widely used activation computer code, ORIGEN2 (see Ref. 3), also requires energy-averaged cross sections. Accordingly, we wrote the ORLIB code to collapse the ACTL library, using the flux as a weighting function. The ORLIB code runs on the LLNL Cray computer rietwork. We have also modified ORIGEN2 to accept the expanded activation libraries produced by ORLIB. The modified ORIGEN2 code, named FORIG, is described in Ref. 4.
\end{abstract}

\section{The Model}

Buildup of a dauginter species can be found from

$\frac{d N_{2}}{d t}=N_{1} \sigma \phi$

where $d N_{2}$ is the production rate of th a daughter, $N_{1}$ is the parent population, $\sigma$ is the cross section, and $\phi$ is the total flux $\left(\mathrm{n} / \mathrm{cm}^{2} / \mathrm{s}\right)$. The cross section is

$\sigma=\frac{\int_{\mathrm{E}} \sigma(\mathrm{E}) \phi(\mathrm{E}) \mathrm{d} \mathrm{E}}{\int_{\mathrm{E}} \phi(\mathrm{E}) \mathrm{dE}}=\frac{\int_{E} \sigma(\mathrm{E}) \phi(\mathrm{E}) \mathrm{dE}}{\phi}$

The energy-dependent flux is obtained fiom the TART neutronics code ${ }^{\overline{5}}$ as a set of neutron-pathlengths-per-source-neutron in 175 energy bins. Then

$\phi(E)=S \frac{\mathbf{L}_{\mathrm{i}}}{\mathrm{VE}_{\mathrm{i}}}$

where $S$ is the number of source neutrons per unit time, $\mathrm{L}_{\mathrm{i}}$ is the total neutron path length in the zone and energy bin, $V$ is the zone volume, and $E_{i}$ is the width of the energy bin. The flux is assumed to be constant at all energies within the energy bin, i. Spatial variation of the parent density and the flux can also be included in the average cross section. In this case,

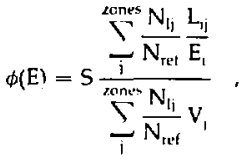

where $N_{1 j}$ is the parent population in zone $i, N_{1}$ in Eq. (1) is replaced by $N_{\mathrm{ret}}$, and $\mathrm{N}_{\mathrm{ref}}=\sum_{i} \mathrm{~N}_{1,}$.

There are two limitations of this procedure: First, the flux must remain constant during the timeframe of the calculation. If the composition, and hence flux spectrum, changes enough to alter the energy-averaged cross sections, the cross sections must be periodically revised. The ORIGEN2 code includes such revisions in its various sets of fission-reactor cross-section libraries. In fusionreactor structure, flux variation is not expected to require frequent recalculation of cross sections. 
The second limitation is due to the spatialaveraging procedure. If activation of daughters is to be considered, the region of the problem must have a constant flux and density, i.e., no spatial averaging is permitted. This is because Eq. (4) must replace $N_{1 j}$ by $N_{2 j}$, the parent of the granddaughter, $N_{3 i}$. However, the spatial distribution of $\mathrm{N}_{2}$ is unknown during the cross-section collapse. Thus, grand-dalighter production will use spatial-weighting fâctors that are appropriate only for daughter production. One method of circumventing this problem is to run separate calculations for each zone. Altematively, a scoping calculation can be used with spatial averaging if the ACTL input is restricted to the original parent nu clides. The reduced input will eliminate most daughter activations. The result can then be compared to a calculation with the full ACTL library. If there is little change, activation of Jaughters is probably insignificant.

\section{The Code}

The ORLIB code includes parts of the OMEGA code ${ }^{6}$ and an undocumented TART postprocesser code. TART is run using sentinel 11 set as the desired name of the TART output data file (which is ther. produced in addition to the high speed file). The TART tally type must be 2 (path length) for ali zones that will be used to collapse the cross sections.

ORLIB requires an input file that specifies the TAK I data file name, the TART zone and tally times, any density-weighting factors, the source sirength, and the nuclides of interest. The input file is described in detail in the next section. ORLIB also requires an ACTL library file.

ORLIB begins by reading the TART output data file and the input file. The density-weighting factors and the energy bin widths are applied to the path-length output, and the quantities $(\phi(E) / S),(\phi / S),\left(\Sigma V_{i}\right)$ are computed. The energy-dependent fluxes are in the form of a histogram. Because duplicate energy coordinates will be eliminated later, the histogram is skewed slightly, as shown in Fig. 1.

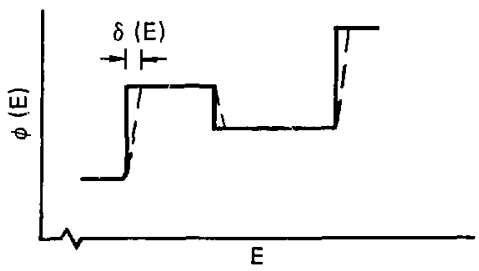

Figure 1. The histogram is shifted slightly to eliminate duplicate energy points. The shift, $\delta(E)$, is the energy coordinate divided by $10^{6}$.
For each reaction of each specified nuclide, the ACTL cross-section data are read (Table 1). These data are in the form of $(E, \sigma)$ points with intermediate values set by linear interpolation. The energy points of the flux and cross sections are merged, and duplicates are eliminated. The flux and cross section is computed at each point by linear interpolation. The numerator of Eq. (2) is then

$\stackrel{\therefore}{i} \sigma(\mathrm{E}) \phi(\mathrm{E}) \mathrm{dE}$.

where the $E$ range of the integral is between the two energy points defining bin $\mathrm{i}$, and $\mathrm{N}$ is the number of bins in the merged energy array.

Table 1. OMEGA reactions.

\begin{tabular}{ll}
\hline $12(n, 2 n)$ & $29(n, 2 n p) \alpha$ \\
$13(n, 3 n)$ & $30(n, \gamma n \alpha)$ \\
$14(n, 4 n)$ & $31(n, 2 n p \alpha) \alpha$ \\
$15(n, f)$ & $37(n, 2 \alpha) \alpha$ \\
$20(n, n p)$ & $38\left(n,{ }^{3} H e \alpha\right) \alpha$ \\
$21(n, p n)$ & $39(n, t p) \alpha$ \\
$22(1,, n d)$ & $40(n, p)$ \\
$23(n, n d u) \alpha$ & $41(n, d)$ \\
$24(n, n t)$ & $42(n, t)$ \\
$25\left(n, n^{3} H e\right)$ & $43(n, t \alpha) \alpha$ \\
$26(n, n \alpha)$ & $44\left(n,{ }^{3} H c\right)$ \\
$27(n, n 2 \alpha) \alpha$ & $45(n, \alpha)$ \\
$28(n, n t \alpha) \alpha$ & $46(n, \gamma)$ \\
\hline
\end{tabular}

OMEGA and ORLIB use the method of Ref. 7 to compute the integral in Eq. (5).

$$
\begin{aligned}
\int_{\mathrm{a}}^{\mathrm{h}} \sigma(\mathrm{E}) \phi(\mathrm{E}) \mathrm{dE} & =\left[\left(\phi_{\mathrm{b}}+\phi_{\mathrm{a}}\right)\left(\sigma_{\mathrm{b}}+\sigma_{\mathrm{a}}\right)\right. \\
& \left.+\frac{1}{3}\left(\phi_{\mathrm{b}}-\phi_{\mathrm{a}}\right)\left(\sigma_{\mathrm{h}}-\phi_{\mathrm{a}}\right)\right] \frac{\left(\mathrm{E}_{\mathrm{b}}-\mathrm{E}_{\mathrm{a}}\right)}{4}
\end{aligned}
$$




\section{ORLIB Input}

The ORLIB execution line is

\section{ORLIB INPT AC820312 $0 / \mathrm{t} v$}

where INPT is the input file name and AC820312 is the ACTL library file. At LLNL the controllee may be outained from J. Blink's take directory.

\section{XPORT}

\section{.RD ,080975:ORIGEN2:ORLIB}

(XPORT is an LLNL file-transport routine.) The ACTL library file name is AC820312 where 820312 is the creation date. To determine the latest library, consult $R$. Howerton's take directory.

\section{XPORT}

\section{.LST .415600:CRACT811}

Select the latest $A C$ date file (AC820312 at the time of this report).

\section{.RD .415600:CRACT811:AC820312}

Readers outside LLNL are invited to contact the author for a copy of the code listing and the ACTL library.

The ORLIB input file has five line types:

\section{Line 1: Format $1 x, 16,1 x, 16,1 x, 12$}

The two ZZAAA numbers are the range of atomic number and mass ( $Z A$ ) over which the available Table 1 reactions in ACTL will be computed. All nuclides between the two bounds will be processed. If $N L \geq 0$, other $Z_{A}$ ranges will be specified in line-type-5 lines at the end of the file. If $N L<0$, this is the only $Z A$ range of interest, and no type 5 lines will be in the file.

Linte 2: Format A8,1x, 13,1x, I3,1x, E11.4
NAME is the TART tape name, LIB is a three-digit number of the output library $(\geq 700$ if the output is to be used in FORIG). $N Z N$ is the number of zones and times to be averaged, and SRCSTR is the neutron source strength in neutrons/second.

Line 3: Format $1 x, 13,1 x, I 3,1 x, E 11.4$

$12345678(1) 2345678(2$ T I $M$ F RAC

TIM is the TART edit time number $(1,2,3$, etc.). ZON is the TART zone number. FRAC is the spatial-weighting factor.

$$
\mathrm{FRAC}=\frac{\rho}{\rho_{\text {ref }}} \frac{\mathrm{mtl} \mathrm{vf}}{\text { ref } v \mathrm{f}},
$$

where $\rho_{\text {ref }}$ and ref vf are the atom density and volume fraction of the reference material. For example, if the reference material is full density graphite occupying 90 vol\% of a zone, and if a particular zone has $50 \%$ density graphite sccupying 80 vol\% of the zone, ther:

$\mathrm{FRAC}=\left(\frac{\mathrm{I}}{2}\right) \frac{0.8}{0.9}$

and the mass of graphite used in Eq. (1) will be

$\mathrm{N}_{1}=\sum_{i}^{\mathrm{ranem}} \mathrm{V}_{1}(\mathrm{vf}) \rho_{1}$.

There is one Line 3 for each zone and time of interest; i.e., NZN lines; NZN $\leq 20$.

Line 4: Format $9 \mathrm{A8}$

A message up to 72 characters long will be printed on the output.

Line 5: Format-same as Line 1

It contains additional $\mathrm{ZA}$ ranges to be processed. The last Line 5 will have NL less than zero. 


\section{ORLIB Output}

ORLIB produces four files: OLEVEL, OACT, OODD, and OGEORGE.

File OLEVEL prints the parent, daughter, and energy level of all reactions involving nuclides not in the ground state. ORLIB nuclide identification is the same as ACTL except the isomer number is added.

\section{ACTL \\ ZZAAA \\ ORLIB ZZAAAI}

The isomer number, 1 , is 0 for the ground state, 1 for the first excited state, etc. Since the ORLIB code dives not check the level, all excited isomers have $I=1$. The OLEVEL file provides the data needed to manually correct the other output files if there are two or more excited states for a given nuclide. The ACTL library AC820312 had no nuclides in this category. Parenthetically, we note that the FORIG and ORIGEN2 codes cannot handle isomer numbers $>1$.

File OACT lists the nuclide identification and 11 reaction cross sections.

ZZAAAI $(n, 2 n)(n, 2 n)$ exc $(n, 3 n)(n, n p)(n, n \alpha)$ $(n, p)\{n, d\}(n, t)(n, \alpha)(n, \gamma)(n, \gamma)$ exc

where "exc" means the daughter is in an excited state. It also prints the source strength, total flux, total zone volume, and material volume. The pasent population, $N_{i}$, in Eqs. (1) and (9) is the material volume multiplied by the product of $\rho_{\text {rer }}$ and $v f_{\text {ref }}$

File OODD lists all othe: reactions.

$\begin{array}{cccc}\text { Parent } & \text { Daughter } & \text { Cross } & \text { OMEGA } \\ \text { LZAAAI } & \text { ZZAAAl } & \text { Section } & \begin{array}{c}\text { Reaction } \\ \text { Code }\end{array}\end{array}$

Files OACT and OODD are renamed TAPE8 and TAPE3, respectively, when they are used as FORIG input.

File OGEORGE is a modification of Files OACT and OODD requested by G. P. Lasche for hand calculations. ${ }^{\text {It }}$ contains the 11 standard reactions, the odd reactions, and the total cross section. Each cross section has been multiplied by a conversion factor

$C=\phi\left(1 \times 10^{-24}\right)$.

where $\phi$ is specified by Eqs. (2) to (4). Thus, these cross sections have units of probability per second. For hand calculations, the "OGEORGE" cross sections are multiplied by the reference parent population, $N_{1}$, in Eq. (9) to obtain the production rates of the daughters in units of atoms/s.

\section{Summary}

A computer code, ORLIB, has been written to average ACTL zross sections in energy and space using a TART calculated space and energy dependent flux and the material composition as weighting factors. The output is suitable for either hand calculations, or input to the FORIG code. If activation of daughters is significant, the code must be run for each individual zone since spatial averaging prior to buildup of the daughters is not appropriate.

\section{Acknowledgments}

The authors are grateful for helpful discussions with J. Hovingh, R. Howerton, G. Lasche, W. Meier, and E. Plechaty, of LLNL; and A. Croff of ORNL. 


\section{References}

1. M. A. Gardner and R. J. Howerton, ACTL: Evaluated Neutron Activation Cross Section Library-Evaltation Techniques and Renction Index, Lawrence Livermore National Laburatory, Livermore, CA, UCRL-50400, Vol. 18 (Oct, 17, 1978).

2. R. J. Howerton, Lawrence Livermore National Laboratory, private communication (Aug. 1981).

3. A. G. Croff, A User's Matual for the ORIGEN2 Computer Code, Oak Ridge National Laboratory, Oak Ridge, TN, ORNL/TM-7175 (July 1980). Alsc published as Radiation Shielding Information Center Report CCC-371.

4. J. A. Blink, FORIG: A Modification of the ORIGEN2 Isotope Generation and Depletion Code for Fusion Problems, Lawrence Livermore National Laboratory, Livermor?, CA, UCRL-53263 (Dec. 1981).

5. E. F, Plechaty and J. R. Kimlinger, TARTNP: A Coupled Neutron-Photon Monte Carlo Transport Code, Lawrence Livermore National Laboratory, Livermore, CA, UCRL-50400, Vol. 14 (July 4, 1976).

6. R. J. Howerton, R. J. Doyas, T. C. Michels, and S. T. Perkins, An Integrated System for Production of Neutronics and Photonics Calculational Constants, Lawience Livermore National Laboratory, Livermore, CA, UCRL-50400, Vol. 4 (April 15, 1971).

7. R. E. Von Holdt and R. J. Howerton, "The Definite Integral of the Product of Linear Functions," Math. Comp. 17, 419 (1963).

8. G. P. Lasche, Upper-Bounded Estimates of Neutron-Induced Radionuclide Populations, Lawrence Livermore National Laboratory, Livermore, CA, UCRL-87255 (1982). 